\title{
Evaluation of polymorphism in ten microsatellite loci in Uruguayan sheep flocks
}

\author{
Ivanna Tomasco, G. Wlasiuk and E.P. Lessa \\ Laboratorio de Evolución, Facultad de Ciencias, Universidad de la República, Montevideo, Uruguay.
}

\begin{abstract}
The allele frequencies of 10 microsatellite loci previously described for sheep as BM1314, BM6526, OarFCB128, OarHH64, OarCP20, OarHH47, OarFCB48, OarHH35, OarHH72 and BM2508 were estimated for the Uruguayan flocks. A representative sample of 101 individuals composed by the two predominant breeds $(76 \%$ Corriedale and $24 \%$ Australian Merino) was used. The sample did not show a significant tendency towards substructuring, in spite of presenting some significantly different allele frequencies between races. The Corriedale sample presents three loci in which the presence of null alleles is possible. The markers were highly variable, showing between 7 and 15 alleles each. The Polymorphism Information Content Index ranged from 0.63 to 0.87 and the Exclusion Probability from 0.39 to 0.75 for a cumulative Exclusion Probability of $99.98 \%$. These results suggest the effectiveness of this set of loci for testing genetic relatedness. This is the first report of microsatellite variation in Corriedale.
\end{abstract}

Key words: sheep, polymorphism, microsatellites.

Received: March 14, 2002; accepted: March 25, 2002.

\section{Introduction}

For every program of breeding and selection, it is desirable to rely on a precise assessment of parentage to assist, for example, in the elimination of undesirable recessive alleles, in early selection for future breeding, or to ensure accurate pedigrees and registration. Microsatellites, segments of the nuclear genome composed of tandem repeat of short-sequence motifs, have become excellent candidate markers for this kind of studies (Queller et al., 1993), since they are numerous, highly variable and easy to score.

A large number of highly polymorphic microsatellites have been characterized and mapped in domestic animals, including sheep, cattle and other ruminants (de Gortari et al., 1997, 1998; Hayes et al., 1996; Jenkings et al., 1997), facilitating the use of these markers in parentage testing. For this kind of test to be acceptable, it must provide a high degree of certainty in the assignment of parentage, which can be evaluated with the Exclusion Probability (Weir, 1996). This requires a previous knowledge of allele frequencies in the population of interest and certainty about the assumptions of the model, like independence of the markers utilized and Hardy-Weinberg equilibrium.

The goals of this study were to analyze the polymorphism of a set of microsatellites previously described as

Send correspondence to Ivanna Tomasco. Facultad de Ciencias, Universidad de la República, Iguá 4225, 11400 Montevideo, Uruguay. E-mail: ivanna@fcien.edu.uy. polymorphic in sheep and to evaluate their usefulness for relatedness testing in Uruguayan sheep flocks.

\section{Material and Methods}

We analyzed ten microsatellite loci in a sample of the Uruguayan flocks considering only pure-breed individuals. The analysis were carried out separately for two subsamples: one of 77 Corriedale individuals from a fair, and another of 24 Australian Merinos from an experimental field (all of them are expected to be unrelated ewes), as well as on the combined dataset. Corriedale, the predominant race in the country, has not been previously characterized using microsatellite markers.

Total DNA extractions were made with SDS/proteinase $\mathrm{K} / \mathrm{NaCl} /$ alcohol precipitation (modified from Miller $e t$ al., 1988) from skin preserved in $95 \%$ ethyl-alcohol. The microsatellite loci OarHH35, OarHH64, OarHH47 (Henry et al., 1993), OarVH72 (Pierson et al., 1993), OarFCB128 (Buchanan and Crawford 1993), OarFCB48 (Buchanan et al., 1994), OarCP20, BM1314, BM6526, (Crawford et al., 1995), and BM2508 (Mulsant et al., 1998) were amplified by PCR (Polymerase Chain Reaction) for all animals; excluding OarHH35 and OarHH64 all of them map on different chromosomes. Amplification was carried out in a total volume of $12.5 \mu \mathrm{L}$ containing the following constituents: $6.25 \mu \mathrm{L}$ of DNA $(0.4 \mu \mathrm{g} / \mathrm{mL})$ used as a template, $1 \mathrm{X}$ Taq Polymerase Buffer, $240 \mu \mathrm{M}$ of each dNTP, $240 \mathrm{nM}$ of 
each primer, 0.5 units of Taq Polymerase and variable concentration of $\mathrm{MgCl}_{2}$ depending on the locus (from 1.5 to $3.0 \mathrm{mM}$ ). PCR amplifications were performed in a Rapidcycler $^{\mathrm{TM}}$ (Idaho Technology) by an initial denaturation of $3 \mathrm{~min}$ at $94{ }^{\circ} \mathrm{C}$, followed by 35 cycles of $45 \mathrm{~s}$ of denaturation at $94^{\circ} \mathrm{C}, 45 \mathrm{~s}$ of annealing at $50^{\circ} \mathrm{C}$ and $45 \mathrm{~s}$ of extension at $72{ }^{\circ} \mathrm{C}$, and a final extension of $7 \mathrm{~min}$ at $72{ }^{\circ} \mathrm{C}$.

The amplified products were electrophoresed in $7 \%$, $0.80 \mathrm{~mm}$ thick denaturing polyacrylamide gels (1000$1200 \mathrm{~V}, 1-2 \mathrm{~h}$ ), and the DNA bands were visualized by silver staining (Sanguinetti et al., 1994). Genotypes were scored by at least two persons independently and the absolute sizes of the alleles were determined in relation to a 10bp DNA size standard (GIBCO BRL).

On the basis of allele and genotype frequencies, Hardy-Weinberg equilibrium and linkage disequilibrium were tested using the software GenePop, version 3.1b (Raymond and Rousset, 1995). A Bonferroni correction was used to adjust significance levels in all tests to an overall $\alpha=0.05$. Observed heterozygosity $\left(\mathrm{H}_{\mathrm{obs}}\right)$, Polymorphism Information Content (PIC, Botstein et al., 1980) and Exclusion Probability assuming that the genotype of the lamb and one of its parents is known (Q, according to Weir, 1996) were calculated.

Table I - Loci, absolute allele sizes in base pairs, allele frequencies, observed heterozygosity $\left(\mathrm{H}_{0 \mathrm{bs}}\right)$, polymorphism information content (PIC), exclusion probability $(\mathrm{Q})$ and number of individuals analyzed per locus and sample. The whole population combines all the Merinos and Corriedales; p-values refer to the comparison of allele frequency distributions between Merino and Corriedale samples: *and NS means significant and non-significant difference, respectively. Markers are followed by $\bullet$ if their absolute allele sizes are not concordant with those reported in the literature.

\begin{tabular}{|c|c|c|c|c|}
\hline \multirow[t]{2}{*}{ Locus } & \multirow{2}{*}{$\begin{array}{l}\text { Allele size } \\
\text { (Bp) }\end{array}$} & \multicolumn{3}{|c|}{ Allele frequencies } \\
\hline & & Whole pop & Merino & Corriedale \\
\hline Sample size (n) & & 101 & 24 & 77 \\
\hline & 117 & 0.010 & 0 & 0.013 \\
\hline \multirow[t]{14}{*}{ OarHH35 } & 121 & 0.094 & 0.229 & 0.052 \\
\hline & 123 & 0.079 & 0.271 & 0.019 \\
\hline & 125 & 0.347 & 0.167 & 0.403 \\
\hline & 127 & 0.109 & 0.229 & 0.071 \\
\hline & 129 & 0.322 & 0.104 & 0.390 \\
\hline & 131 & 0.010 & 0 & 0.013 \\
\hline & 133 & 0.005 & 0 & 0.006 \\
\hline & 135 & 0.015 & 0 & 0.019 \\
\hline & 137 & 0.010 & 0 & 0.013 \\
\hline & & p-value & $0.00001 *$ & \\
\hline & $\mathrm{H}_{\mathrm{obs}}$ & 0.72 & 0.77 & 0.70 \\
\hline & PIC & 0.71 & 0.75 & 0.62 \\
\hline & Q & 0.53 & 0.57 & 0.43 \\
\hline & 130 & 0.025 & 0 & 0.032 \\
\hline
\end{tabular}

The software Structure (Pritchard et al., 2000) was used in order to assess levels of subdivision between two races (10,000 Burn-in period, 10,000 MCMC repetitions, and default parameters).

\section{Results}

Allele length in base pairs, allele frequencies, $\mathrm{H}_{\mathrm{obs}}$, PIC, Q and the number of individuals analyzed per locus are given in Table I. All loci were highly polymorphic, showing between 7 and 15 alleles, PICs between 0.63 and 0.87 and $\mathrm{H}_{\mathrm{obs}}$ in the range of 0.62 to 0.84 for the whole sample. The two loci that map on the same chromosome (OarHH35 and OarHH64) did not show a significant linkage $(p>0.373, \mathrm{SE}=0.052)$.

The loci OarHH35, OarHH64 and OarFCB128 showed significant departures from Hardy-Weinberg equilibrium in the whole population and in the Corriedale sample (Table II). With the exception of OarFCB128, OarVH72 and BM6526, all loci presented significant differences in allele frequencies between Merino and Corriedale samples. Some alleles were much more frequent in one race than in the other (e.g., allele 123 in OarHH35 and 146 in OarHH47, which are 14 and 8 times more frequent, respectively in Merino than in Corriedale, or allele 75 in

Table I (cont.)

\begin{tabular}{|c|c|c|c|c|}
\hline \multirow[t]{2}{*}{ Locus } & \multirow{2}{*}{$\begin{array}{l}\text { Allele size } \\
\text { (Bp) }\end{array}$} & \multicolumn{3}{|c|}{ Allele frequencies } \\
\hline & & Whole pop & Merino & Corriedale \\
\hline Sample size (n) & & 101 & 24 & 77 \\
\hline \multirow[t]{12}{*}{ OarHH64 } & 132 & 0.060 & 0.043 & 0.065 \\
\hline & 136 & 0.015 & 0 & 0.019 \\
\hline & 138 & 0.299 & 0.191 & 0.331 \\
\hline & 140 & 0.055 & 0 & 0.071 \\
\hline & 142 & 0.109 & 0.277 & 0.058 \\
\hline & 144 & 0.199 & 0.191 & 0.201 \\
\hline & 146 & 0.239 & 0.298 & 0.221 \\
\hline & & $\mathrm{p}$-value & $0.00260 *$ & \\
\hline & $\mathrm{H}_{\mathrm{obs}}$ & 0.69 & 0.83 & 0.65 \\
\hline & PIC & 0.77 & 0.72 & 0.76 \\
\hline & Q & 0.59 & 0.44 & 0.54 \\
\hline & 126 & 0.020 & 0 & 0.026 \\
\hline \multirow[t]{9}{*}{ OarHH47 } & 128 & 0.005 & 0.021 & 0 \\
\hline & 132 & 0.005 & 0 & 0.007 \\
\hline & 134 & 0.085 & 0 & 0.112 \\
\hline & 136 & 0.095 & 0.063 & 0.105 \\
\hline & 138 & 0.055 & 0.104 & 0.039 \\
\hline & 140 & 0.485 & 0.583 & 0.454 \\
\hline & 142 & 0.110 & 0 & 0.145 \\
\hline & 144 & 0.025 & 0 & 0.033 \\
\hline & 146 & 0.035 & 0.104 & 0.013 \\
\hline
\end{tabular}


Table I (cont.)

\begin{tabular}{|c|c|c|c|c|}
\hline \multirow[t]{2}{*}{ Locus } & \multirow{2}{*}{$\begin{array}{l}\text { Allele size } \\
\quad(\mathrm{Bp})\end{array}$} & \multicolumn{3}{|c|}{ Allele frequencies } \\
\hline & & Whole pop & Merino & Corriedale \\
\hline \multirow[t]{8}{*}{ Sample size (n) } & & 101 & 24 & 77 \\
\hline & 148 & 0.060 & 0.083 & 0.053 \\
\hline & 150 & 0.020 & 0.042 & 0.013 \\
\hline & & p-value & $0.00002 *$ & \\
\hline & $\mathrm{H}_{\mathrm{obs}}$ & 0.66 & 0.54 & 0.70 \\
\hline & PIC & 0.72 & 0.60 & 0.72 \\
\hline & Q & 0.55 & 0.43 & 0.56 \\
\hline & 142 & 0.010 & 0 & 0.013 \\
\hline OarFCB48 & 144 & 0.120 & 0.261 & 0.078 \\
\hline \multirow[t]{18}{*}{ - } & 146 & 0.025 & 0.022 & 0.026 \\
\hline & 148 & 0.370 & 0.217 & 0.416 \\
\hline & 150 & 0.275 & 0.109 & 0.325 \\
\hline & 152 & 0.015 & 0.043 & 0.006 \\
\hline & 154 & 0.040 & 0.109 & 0.019 \\
\hline & 156 & 0.070 & 0.152 & 0.045 \\
\hline & 158 & 0.005 & 0 & 0.006 \\
\hline & 160 & 0.010 & 0 & 0.013 \\
\hline & 162 & 0.015 & 0.065 & 0 \\
\hline & 164 & 0.010 & 0 & 0.013 \\
\hline & 166 & 0.005 & 0 & 0.006 \\
\hline & 168 & 0.025 & 0.022 & 0.026 \\
\hline & 170 & 0.005 & 0 & 0.006 \\
\hline & & p-value & $0.0001 *$ & \\
\hline & $\mathrm{H}_{\mathrm{obs}}$ & 0.73 & 0.96 & 0.66 \\
\hline & PIC & 0.73 & 0.79 & 0.67 \\
\hline & Q & 0.55 & 0.67 & 0.46 \\
\hline & 100 & 0.122 & 0 & 0.149 \\
\hline OarFCB128 & 112 & 0.058 & 0.114 & 0.045 \\
\hline \multirow[t]{11}{*}{$\bullet$} & 114 & 0.243 & 0.286 & 0.234 \\
\hline & 120 & 0.005 & 0.029 & 0 \\
\hline & 122 & 0.048 & 0 & 0.058 \\
\hline & 124 & 0.196 & 0.143 & 0.208 \\
\hline & 126 & 0.259 & 0.400 & 0.227 \\
\hline & 128 & 0.069 & 0.029 & 0.078 \\
\hline & & p-value & $0.00732 \mathrm{NS}$ & \\
\hline & $\mathrm{H}_{\mathrm{obs}}$ & 0.68 & 0.59 & 0.70 \\
\hline & PIC & 0.78 & 0.68 & 0.79 \\
\hline & Q & 0.63 & 0.45 & 0.63 \\
\hline & 127 & 0.051 & 0.068 & 0.046 \\
\hline \multirow[t]{6}{*}{ OarVH72 } & 129 & 0.454 & 0.318 & 0.493 \\
\hline & 131 & 0.036 & 0.068 & 0.026 \\
\hline & 133 & 0.276 & 0.409 & 0.237 \\
\hline & 137 & 0.036 & 0 & 0.046 \\
\hline & 139 & 0.102 & 0.114 & 0.099 \\
\hline & 141 & 0.026 & 0 & 0.033 \\
\hline
\end{tabular}

Table I (cont.)

\begin{tabular}{|c|c|c|c|c|}
\hline \multirow[t]{2}{*}{ Locus } & \multirow{2}{*}{$\begin{array}{l}\text { Allele size } \\
\text { (Bp) }\end{array}$} & \multicolumn{3}{|c|}{ Allele frequencies } \\
\hline & & Whole pop & Merino & Corriedale \\
\hline \multirow[t]{7}{*}{ Sample size (n) } & & 101 & 24 & 77 \\
\hline & 143 & 0.020 & 0.023 & 0.020 \\
\hline & & $\mathrm{p}$-value & $0.09948 \mathrm{NS}$ & \\
\hline & $\mathrm{H}_{\mathrm{obs}}$ & 0.62 & 0.64 & 0.62 \\
\hline & PIC & 0.66 & 0.66 & 0.65 \\
\hline & Q & 0.48 & 0.47 & 0.47 \\
\hline & 71 & 0.005 & 0 & 0.006 \\
\hline \multirow[t]{14}{*}{ OarCP20 } & 73 & 0.337 & 0.313 & 0.344 \\
\hline & 75 & 0.119 & 0.021 & 0.149 \\
\hline & 77 & 0.114 & 0.125 & 0.110 \\
\hline & 79 & 0.129 & 0.104 & 0.136 \\
\hline & 81 & 0.015 & 0.021 & 0.013 \\
\hline & 83 & 0.238 & 0.271 & 0.227 \\
\hline & 85 & 0.035 & 0.146 & 0 \\
\hline & 87 & 0.005 & 0 & 0.006 \\
\hline & 89 & 0.005 & 0 & 0.006 \\
\hline & & $\mathrm{p}$-value & $0.00070^{*}$ & \\
\hline & $\mathrm{H}_{\mathrm{obs}}$ & 0.72 & 0.79 & 0.70 \\
\hline & PIC & 0.76 & 0.72 & 0.75 \\
\hline & Q & 0.59 & 0.58 & 0.57 \\
\hline & 140 & 0.213 & 0.159 & 0.321 \\
\hline \multirow[t]{17}{*}{ BM1314 } & 150 & 0.075 & 0 & 0.100 \\
\hline & 155 & 0.086 & 0.045 & 0.100 \\
\hline & 157 & 0.144 & 0.182 & 0.131 \\
\hline & 159 & 0.149 & 0.364 & 0.077 \\
\hline & 161 & 0.057 & 0.045 & 0.062 \\
\hline & 163 & 0.034 & 0 & 0.046 \\
\hline & 165 & 0.023 & 0.023 & 0.023 \\
\hline & 197 & 0.023 & 0 & 0.031 \\
\hline & 169 & 0.006 & 0 & 0.008 \\
\hline & 171 & 0.069 & 0.045 & 0.077 \\
\hline & 173 & 0.098 & 0.114 & 0.092 \\
\hline & 175 & 0.023 & 0.023 & 0.023 \\
\hline & & $\mathrm{p}$-value & $0.00444 *$ & \\
\hline & $\mathrm{H}_{\mathrm{obs}}$ & 0.84 & 0.77 & 0.86 \\
\hline & PIC & 0.87 & 0.76 & 0.81 \\
\hline & Q & 0.76 & 0.61 & 0.77 \\
\hline & 124 & 0.016 & 0.027 & 0.013 \\
\hline \multirow[t]{7}{*}{ BM6526 } & 126 & 0.026 & 0.054 & 0.019 \\
\hline & 128 & 0.005 & 0 & 0.006 \\
\hline & 130 & 0.016 & 0.027 & 0.013 \\
\hline & 132 & 0.068 & 0 & 0.084 \\
\hline & 134 & 0.204 & 0.162 & 0.214 \\
\hline & 136 & 0.079 & 0 & 0.097 \\
\hline & 138 & 0.183 & 0.108 & 0.201 \\
\hline
\end{tabular}




\begin{tabular}{|c|c|c|c|c|}
\hline \multirow[t]{2}{*}{ Locus } & \multirow{2}{*}{$\begin{array}{l}\text { Allele size } \\
\quad(\mathrm{Bp})\end{array}$} & \multicolumn{3}{|c|}{ Allele frequencies } \\
\hline & & Whole pop & Merino & Corriedale \\
\hline \multirow[t]{11}{*}{ Sample size (n) } & & 101 & 24 & 77 \\
\hline & 140 & 0.079 & 0.162 & 0.058 \\
\hline & 142 & 0.298 & 0.459 & 0.260 \\
\hline & 144 & 0.010 & 0 & 0.013 \\
\hline & 146 & 0.005 & 0 & 0.006 \\
\hline & 148 & 0.010 & 0 & 0.013 \\
\hline & & p-value & $0.02158 \mathrm{NS}$ & \\
\hline & $\mathrm{H}_{\mathrm{obs}}$ & 0.75 & 0.67 & 0.77 \\
\hline & PIC & 0.80 & 0.70 & 0.80 \\
\hline & Q & 0.64 & 0.51 & 0.65 \\
\hline & 88 & 0.005 & 0 & 0.007 \\
\hline \multirow[t]{10}{*}{ BM2508 } & 92 & 0.351 & 0.125 & 0.412 \\
\hline & 100 & 0.138 & 0.150 & 0.135 \\
\hline & 104 & 0.016 & 0.075 & 0 \\
\hline & 112 & 0.410 & 0.650 & 0.345 \\
\hline & 114 & 0.043 & 0 & 0.054 \\
\hline & 116 & 0.037 & 0 & 0.047 \\
\hline & & $\mathrm{p}$-value & $0.00001^{*}$ & \\
\hline & $\mathrm{H}_{\mathrm{obs}}$ & 0.65 & 0.50 & 0.69 \\
\hline & PIC & 0.63 & 0.50 & 0.63 \\
\hline & Q & 0.39 & 0.32 & 0.44 \\
\hline
\end{tabular}

OarCP20 which is seven times more frequent in Corriedale than in Merino). Other alleles were found only in one sample, and sometimes at high frequency (e.g., alleles 134 and 142 of OarHH47 in Corriedale, and allele 85 of OarCP20 in Merino). In several cases, these frequencies differed from those reported in the literature (see Henry et al., 1993, for OarHH47, OarHH35, OarHH64; Buchanan and Crawford
1993, for OarFCB128; Ede et al., 1995, for OarCP20; and Buchanan et al., 1994, for OarFCB48). There were also differences in one base pair between absolute allele sizes reported for OarFCB48 and OarFCB128 and our observations but it was easy to resolve the correspondences on the basis of allele frequencies. Direct comparisons would be needed to assess the reason for this discrepancy.

Analyses of subdivision using the software Structure (Pritchard et al., 2000) did not find a significant tendency towards substructuring in the sample (data not shown), in spite of the presence of two breeds.

\section{Discussion}

The loci used in the analysis are effectively independent, since the only two that map on the same chromosome (OarHH35 and OarHH64) did not show significant linkage disequilibrium. The departures from Hardy-Weinberg equilibrium shown by OarHH35, OarHH64 and OarFCB128 in the Corriedale sample were probably the cause of the departure in the whole population. This could be due to the presence of null alleles in these loci in Corriedale or be the consequence of several years of intensive selection on these breeds (Usha et al., 1995). To avoid this problem, these loci can be excluded from parentage analysis and the cumulative Q would vary from $99.99 \%$ to $99.77 \%$ and from $99.98 \%$ to $99.75 \%$ for the whole population and the Corriedale stock, respectively. For Merinos, the cumulative Q with ten loci was $99.93 \%$.

In spite of the presence of several differences in allele frequencies between races, these were insufficient to result in substantial separation between these breeds. These two races are known to be related, since Corriedale was originated from crosses between pure Merino and Lincoln sheep (Majala, 1997), so this result is not entirely surprising. Coupled with the fact that most of Uruguayan sheep are not completely pure, these observations suggest that allele

Table II - Observed p-values and standard errors (SE) of Hardy-Weinberg exact tests for heterozygote deficit.

\begin{tabular}{lllllll}
\hline & \multicolumn{3}{c}{$\mathrm{p}$ - value and SE } \\
\cline { 2 - 7 } Locus & \multicolumn{2}{c}{ Whole pop } & \multicolumn{3}{c}{ Merino } & \multicolumn{3}{c}{ Corriedale } \\
\hline OarHH35 & $0.0022^{*}$ & 0.0015 & 0.4380 & 0.0110 & $0.0011^{*}$ & 0.0008 \\
OarHH64 & $0.0000^{*}$ & 0.0000 & 0.7105 & 0.0102 & $0.0000^{*}$ & 0.0000 \\
OarHH47 & 0.0243 & 0.0091 & 0.0319 & 0.0076 & 0.0855 & 0.0180 \\
OarFCB48 & 0.5571 & 0.0555 & 0.9664 & 0.0075 & 0.2182 & 0.0422 \\
OarFCB128 & $0.0003^{*}$ & 0.0003 & 0.0478 & 0.0052 & $0.0016^{*}$ & 0.0010 \\
OarVH72 & 0.2808 & 0.0219 & 0.3717 & 0.0161 & 0.2687 & 0.0212 \\
OarCP20 & 0.0917 & 0.0177 & 0.5716 & 0.0236 & 0.0319 & 0.0111 \\
BM1314 & 0.2119 & 0.0294 & 0.1718 & 0.0233 & 0.3823 & 0.0386 \\
BM6526 & 0.0658 & 0.0210 & 0.0835 & 0.0115 & 0.1427 & 0.0287 \\
BM2508 & 0.2454 & 0.0217 & 0.1807 & - & 0.6460 & 0.0252 \\
\hline
\end{tabular}

*Means significant departures from equilibrium, after a Bonferroni correction (corrected $\alpha=0.005$ ). See text. 
frequency estimations derived from the whole sample may be sufficient for future applications in relatedness tests. Also, such tests may exclude the loci suspected to have null alleles without a marked reduction in the probability of exclusion.

The fact that estimated frequencies differed from those reported in the literature in most loci analyzed, stresses the importance of independent estimates for different regions and races.

In sum, these results indicate that the selected system of markers is highly effective for relatedness studies, with a Probability of Exclusion greater than $99.9 \%$ considering all loci. The significance of this report, the first of this kind in the country, is that it offers interesting perspectives for the incorporation of molecular genetic techniques to animal breeding in Uruguay. In addition, our results represent an original contribution of allele frequencies for regional Corriedale and Australian Merino races.

\section{Acknowledgments}

We are grateful to Jaime Mendoza, Roberto Cardellino and Mario Azzarini, from SUL (Secretariado Uruguayo de la Lana) for encouragement and collaboration in the field work, and to CSIC (Comisión Sectorial de Investigación Científica de la Universidad de la República) for financial support of this work.

\section{References}

Botstein D, White RL, Skolnick M and David R (1980) Construction of a genetic linkage map in man using restriction fragment length polymorphisms. Am. J. Hum. Genet. 32:314-331.

Buchanan F and Crawford AM (1993) Ovine microsatellites at the OarFCB11, OarFCB128, OarFCB193, OarFCB266 and OarFCB304 loci. Anim. Genet. 24:145.

Buchanan FC, Galloway SM and Crawford AM (1994) Ovine microsatellites at the OarFCB5, OarFCB19, OarFCB20, OarFCB48, OarFCB129 and OarFCB226 loci. Anim. Genet. 25:60.

Crawford AM, Dodds KG, Ede AJ, Pierson CA, Montgomery GW, Garmonsway HG, Beattie AE, Davies K, Maddox JF, Kappes SW, et al. (1995). An autosomal genetic linkage map of the sheep genome. Genetics. 140:703-724.

de Gortari MJ, Freking BA, Kappes SM, Leymaster KA, Crawford AM, Stone RT and Beattie CW (1997) Extensive genomic conservation of cattle microsatellite heterozygosity in sheep. Animal. Genetics. 28:274-290. de Gortari MJ, Freking BA, Cuthbertson RP, Kappes SM, Keele JW, Stone RT, Leymaster KA, Dodds KG, Crawford AM and Beattie CW (1998) A second-generation linkage map of the sheep genome. Mamm. Genome. 9:204-209.

Ede AJ, Pierson CA and Crawford AM (1995) Ovine microsatellites at the OarCP9, OarCP16, OarCP20, OarCP21, OarCP23 and OarCP26 loci. Anim. Genet. 26:129-130.

Henry HM, Penty JM, Pierson CA and Crawford AM (1993) Ovine microsatellites at the OarHH35, OarHH41, OarHH44, OarHH47and OarHH64 loci. Anim. Genet. 24:222.

Hayes H, Le Chalony C, Goubin G, Mercier D, Payen E, Bignon C and Kohno K (1996) Localization of ZNF164, ZNF146, GGTA1, SOX2, PRLR and EEF2 on homologous cattle, sheep and goat chromosomes by fluorescent in situ hybridization and comparison with the human gene map. Cytogenet. Cell Genet. 72:342-346.

Jenkins ZA, Henry HM, Galloway SM, Dodds KG and Montgomery GW (1997) Comparative linkage mapping of genes on sheep chromosome 3 provides evidence of chromosomal rearrangements in the evolution of Bovidae. Cytogenet. Cell Genet. 78:272-274.

Majala K (1997) Genetics aspects of domestication, common breeds and their origin. In: Piper L and Rubinsky A (eds) The genetics of sheep. CAB International, U.K , pp. 13-50.

Miller SA, Dikes DD and Polesky HH (1988) A simple salting procedure for extracting DNA from human nucleated cells. Nucleic Acids Res. 16:215.

Mulsant P, Schibler L, Lecerf F, Riquet J, Chitour N, Eggen A, Cribiu EP, Lanneluc I and Elsen JM (1998) Regional mapping of the FecB (Booroola) region of sheep chromosome 6 . Anim. Genet. 29:36-37.

Pierson CA, Hanrahan V, Ede AJ and Crawford AM (1993) Ovine microsatellites at the OarVH34, OarVH58, OarVH61 and OarVH72 loci. Anim. Genet. 24:224.

Pritchard JK, Stephens M and Donnelly P (2000) Inference of population structure using multilocus genotype data. Genetics. 155:945-959.

Queller DC, Strassmann JE and Hughes CR (1993) Microsatellites and Kinship. Tree. 8:285-288.

Raymond M and Rousset F (1995) GenePop (version 1.2). Population genetics software for exact test and ecumenisism. J. Hered. 86:248-249.

Sanguinetti CJ, Neto ED and Simpson AJG (1994) Rapid silver staining and recovery of PCR products separated on polyacrilamide gels. Biotechniques. 17:915-918.

Usha AP, Simpson SO and Williams JL (1995) Probability of random sire exclusion using microsatellite markers for parentage verification. Anim. Genet. 26:155-161.

Weir BS (1996) Genetic data analysis II. Sinauer Associates, Inc. Publishers. Sunderland, Massachusetts, 445 pp. 